BULL. AUSTRAL. MATH. SOC.

VOL. $13(1975), 321-323$.

\title{
Centralizers involving Mathieu groups
}

\section{M.J. Curran}

A simple group $G$ cannot contain a central involution $t$ with $C_{G}(t)=\langle t\rangle \times M$, where $M$ is isomorphic to a simple Mathieu group.

There have been investigations of groups $G$ which contain a central involution $t$ such that $C(t)$ has the form $\langle t\rangle \times M$ where $M$ is a simple non-abelian group ([1], [4], [5]). In this note, the case where $M$ is isomorphic to a Mathieu group is considered.

THEOREM. Let $G$ be a finite group with a central involution $t$ such that $C(t)=(t) \times M$ where $M$ is isomorphic to any one of the simple Mathieu groups. Then $G=O(G) \cdot C(t)$.

Proof. Since $t$ is central, $C(t)$ contains an $S_{2}$-subgroup $S$ of $G$ with $t \in Z(S)$. We show $t$ is not conjugate in $G$ to any other involution in $S$ and the result then follows by Glauberman's $Z^{*}$-theorem ([2]).

(a) First suppose $M$ is isomorphic to $M_{11}, M_{22}$, or $M_{23}$. Then $M$ has only one class of involutions with representative $z$ say. Since $z$ is the square of an element of order 4 in $M$, it follows from the structure of $C(t)$ that $t$ cannot be conjugate to $z$ in $G$. If $t \sim t z$ in $G$, say $(t z)^{a}=t$ for some $a \in G$, then $t \in C(t z)$. So $t^{a} \in C(t)$ and without loss of generality we may suppose $t^{a}=t z$. Thus $a$

Received I July 1975. 
normalizes $C(t, z)$ with $a^{2} \in C(t, z)$, so $|\langle C(t, z), a\rangle|=2|C(t, z)|$. This contradicts the fact that $C(t, z)$ contains a $S_{2}$-subgroup of $G$.

Thus $t$ is not conjugate to $t z$ in $G$.

(b) Suppose $M$ is isomorphic to $M_{12}$ or $M_{24}$. Then $M$ has two classes of involutions; a central class with representative $z$ say (which is again the square of an element of order 4 in $M$ ), and a non-central class with representative $y$ say. (When $M \approx M_{12}$ take $z=\pi$ and $y=\tau$ in [6], and when $M \approx M_{24}$ take $z=z_{1}$ and $y=z_{3} \pi$ in [3].)

(i) As in (a) above, $t$ cannot be conjugate to $z$ or $t z$.

(ii) Suppose $y \sim t$ in $G$, say $y^{b}=t$ for $b \in G$. Then $t^{b} \in C(t)$ and we may suppose $t^{b}=y$ or $t y$. In either case, $b$ centralizes $C(t, y)$.

Now $C(t, y)=\langle t\rangle \times C_{M}(y)$ and $S=\langle t\rangle \times C_{M}(y, z)$ is an $S_{2}$-subgroup of $c(t, y)$ (see [3], [6]). Since $s^{b}$ is also an $s_{2}$-subgroup of $C(t, y), s^{b}=s^{g}$ for some $g \in C(t, y)$.

Thus $S^{b}=\langle t\rangle \times C_{M}\left(y, z^{m}\right)$ where $g=t^{\alpha} m ; \alpha=0$ or $\alpha=1$ and $m \in M$. However $S^{\prime}=\langle z\rangle$ when $M \approx M_{12}$, and $S^{\prime \prime}=\langle z\rangle$ when $M \approx M_{24}$ (Lemma 1 in [6], Lemma 2.3 in [3]); so $b$ conjugates $\langle z\rangle$ to $\langle z\rangle$. Replacing $b$ by $c=b m^{-1}$ we have $y^{c}=t$ and $z^{c}=z$.

However a calculation shows $y z \sim y$ in $M$. Conjugating this relation by $c$ we have $t z \sim t$ in $G$, which contradicts (i) above. Thus $y$ is not conjugate to $t$ in $G$.

(iii) Finally suppose $t y \sim t$ in $G$, say $(t y)^{d}=t$ for some $d \in G$. Then, as above, we may assume $t^{d}=t y$ and further, as in (ii), we may find an $e \in G$ such that $t^{e}=t y$ and $z^{e}=z$. Thus $(t z)^{e}=t y z \sim t y$ in $G$, again contradicting (i). So $t$ is not conjugate to $t y$ in $G$ and the result now follows from Glauberman's $Z^{*}$-theorem. 


\section{References}

[1] M.J. Curran, "Groups with decomposable involution centralizers", submitted.

[2] George Glauberman, "Central elements in core-free groups", J. Algebra 4 (1966), 403-420.

[3] Dieter Held, "The simple groups related to $M_{24}$ ", J. AZgebra 13 (1969), 253-296.

[4] Zvonimir Janko, "A new finite simple group with abelian Sylow 2-subgroups and its characterization", J. Algebra 3 (1966), $147-186$.

[5] Zvonimir Janko and John G. Thompson, "On a class of finite simple groups of Ree", J. AZgebra 4 (1966), 274-292.

[6] W.J. Wong, "A characterization of the Mathieu group $M_{12}$ ", Math. Z. $84(1964), 378-388$.

Department of Mathematics,

University of Auckland,

Auckland,

New Zealand. 